\title{
Alltagszustand „Risikobereitschaft“? Ethnografisch-kulturwissenschaftliches Wissen über Risiko in der Kreativwirtschaft
}

ALEXA FÄRBER

The culture/creative industries are a high risk sector with extremely variable market chances. This is true for global culture/film productions as well as for local culture/creative scenes or SMEs acting on a regional level.

(Fesel/Söndermann 2007: 25).

Die Studie der UNESO zur Kultur- und Kreativwirtschaft in Deutschland zeichnet ein insgesamt positives Bild dieses Sektors, ist hier doch in den vergangenen Jahren ein Wirtschaftsbereich entstanden, der nicht nur wirtschaftliche Erträge erbringt, sondern auch dort, wo sich Kreative sichtbar ansiedeln, einen Imagegewinn verspricht. Neben dem ökonomischen und symbolischen Kapital, das sich hier akkumulieren lässt, bringt dieser Sektor jedoch auch die genannten strukturellen Unwägbarkeiten mit sich: Wenn Kreativwirtschaft mit "hohem Risiko“ verbunden ist, dann baut sie offenbar auf eine gesteigerte Risikobereitschaft. Die Charakterisierung der „kreativen Klasse“, wie sie in Anlehnung an Richard Florida äußerst populär geworden ist, unterstreicht diese Annahme, indem Risikobereitschaft als einer der gemeinsamen Nenner der vielfältigen ProtagonistInnen darstellt wird (Florida 2002). Risiko und die Bereitschaft, es einzugehen, müssen indes, und das interessiert an dieser Stelle, als hochgradig individualisiert angesehen werden und zwar für ein breites Spektrum an Akteuren. 
Das Forschungsfeld „Kreativwirtschaft“ hat sich in den vergangenen zehn Jahren vor allem als ein multi-disziplinäres herausgebildet, zumal dann, wenn es mit Fragen der Stadtforschung verkoppelt wird: Sei es die in der geschichtswissenschaftlichen Forschung angestoßene Frage nach historischen Formen kreativer Milieus (Heßler/Zimmermann 2008), oder die in Soziologie, Wirtschafts- und Kulturgeografie durchgeführten Untersuchungen zu gegenwärtigen Formen der Clusterbildung (Krätke 2002; Scott 2000) und der Genese spezifischer Stadtentwicklungsparadigmen der „kreativen Stadt“ (O’Connor 2005; Heßler 2007). Ethnografische Studien haben sich zudem der genauen Beobachtung von Milieus gewidmet, von professionellen und subkulturellen kreativen Praktiken und ihrer Materialisierung im Stadtraum (Lange 2007; Schwanhäußer 2008; Vogt 2005).

Die Spezifik ethnografisch arbeitender Kulturwissenschaften liegt in der Generierung von Material durch Teilnehmende Beobachtung: Diese in die Tiefe einzelner Fallbeispiele gehende Perspektive passt sich im Forschungsprozess den Entwicklungen des Forschungsfeldes an, sie ist multi-lokal und geht vergleichend vor. Die Analyse des auf diese Weise selbst generierten qualitativen Materials bezieht quantitative Daten und diskursanalytische Textauswertung ein, überprüft inwiefern das Material zum gewählten disziplinären Theorieansatz beiträgt und unterzieht diesen Erkenntnisprozess einer reflexiven Perspektive (Schmidt-Lauber 2007). Im interdisziplinären Stadtforschungszusammenhang widmet sich eine solche theoretisch informierte und reflexive Mikro-/Tiefenperspektive vor allem der Alltagskultur (Kokot 2000). Für das Verständnis der Bedeutung von Risiko in der Kreativwirtschaft und des Umgangs mit Risiko ist eine solche Perspektive deshalb relevant, weil sie Attraktion und Bürde risikoreicher Praktiken im Alltag nachzeichnen und daraus Wissen über mögliche Formen kollektiver Absicherung bereitstellen kann. Dieser Beitrag stellt nur einen ersten Schritt in diese Richtung dar und hat zum Ziel, die zu untersuchenden Akteursgruppen, Praxisformen und stadtspezifischen Ausformungen von Risiko in der Kreativwirtschaft zu umreißen.

\section{Risiko als Untersuchungsgegenstand}

„Risiko“ zählt sicherlich zu den Schlüsselbegriffen der spätmodernen Gesellschaftsanalyse. Ulrich Beck hat erstmals Mitte der 1980er Jahre und jüngst in seiner deutschen Fassung der ,Weltrisikogesellschaft" (2007) die überindividuellen Dimensionen globaler Risiken in ihrer gesellschaftlichen Ausformung untersucht und zahlreiche Studien in die- 
sem Zusammenhang angestoßen. Für die Soziologie der Risikogesellschaft gilt Risiko als eine Begleiterscheinung nicht etwa des Versagens, sondern des Erfolgs der kapitalistischen Gesellschaftssysteme. Die Risikogesellschaft zeichnet sich nach Beck durch die zunehmende „Bedeutung von Entscheidung, Unsicherheit und Wahrscheinlichkeit“" aus (Beck 2007: 19). Damit sind drei elementare Bestandteile von Risiko benannt, denen eine ethnografisch-kulturwissenschaftliche Perspektive nachgehen sollte, um die kulturellen Formen von Risiko akteurs-, praxis- und stadtorientiert zu untersuchen.

Was die Soziologie der Risikogesellschaft anhand konkreter Ereignisse und ihrer gesellschaftlichen Verarbeitung untersucht, interessiert die Gouvernementalitätsstudien mit Blick auf Subjektkonstruktionen. Ausgehend von den spezifischen, diskursiv vermittelten Anforderungen an spätmoderne Subjekte zeichnen diese Studien eine Risikologik nach, die an sich nicht neu ist, aber ihren Adressatenkreis erheblich erweitert hat: Ein „Modell der Konstruktion und Bearbeitung von Risiken“ greift auf „,nahezu alle gesellschaftlichen Bereiche“ über, so Henning SchmidtSemisch. Dieses Modell hat ,die Eigenverantwortung der Subjekte zu ihrem Ziel und ihrer Voraussetzung zugleich, gelten soll sie im Guten wie im Schlechten. Indem Autonomie und Responsibilisierung zusammenfallen, die Subjekte also stets auch mit der Unsicherheit des selbst verschuldeten Scheiterns leben müssen, schreibt sich die marktwirtschaftliche Risikologik auf ambivalente Art und Weise in Befindlichkeit, Handeln und Denken der Individuen ein“" (Schmidt-Semisch 2004: 223f.).

Dieses marktförmige und subjektivierte Risiko richtet sich an ein „,unternehmerisches Selbst“ und lässt sich in Anlehnung an Klassiker der Wirtschaftswissenschaften in „reine Ungewissheit“ und „kalkulierbare Risiken“ unterscheiden: „Ohne rationale Planung und Kontrolle kommt keine Unternehmung aus, aber zum Unternehmer im Sinne Knights wird nur, wer immer wieder den Schritt hinaus ins Ungewisse wagt.“ (Bröckling 2007: 119)

Es sind die subjektkonstituierenden Anforderungen und Freiheitsversprechen, nicht die sich daran abarbeitenden konkreten und beobachtbaren Praktiken, die in den Gouvernementalitätsstudien im Vordergrund stehen und einer kritischen Analyse unterzogen werden. Kreativität gilt dabei als ein zentraler Imperativ, Risiko als eine Begleiterschei- 
nung. ${ }^{1}$ Dies hat auch die 2003 vom Bundestag als Enquete-Kommission eingesetzte Expertengruppe „Kultur in Deutschland“ erkannt, die Künstler als die „Prototypen neuer Selbst- und Einzelunternehmer“ identifiziert, die ,sich durch Flexibilität, Mobilität, Parallelarbeit und ein hohes $\mathrm{Maß}$ an Risikobereitschaft" auszeichnen (Deutscher Bundestag 2007: 290). Gleichzeitig muss sie aber den geringen Kenntnisstand über die Bedeutung dieses „Prototyps“ für die Alltagspraxis feststellen. Diese „derzeit noch nicht präzise beschreibbare Mischung aus wirtschaftlichen Armutsrisiken und subjektiven Autonomiegewinnen“ (ebd.: 295) bietet aber eine Reihe von Beobachtungspunkten für die ethnografisch-kulturwissenschaftliche Forschung.

\section{Ethnografisch-kulturwissenschaftliche Perspektiven auf Risiko und Kreativwirtschaft}

Den Beitrag ethnografischer Untersuchungen in der ,gegenwärtigen Krise der Arbeitsgesellschaft und des Sozialstaates“ sieht Bernd Jürgen Warneken weniger in der realistischen Darstellung der ökonomischen Situation und Bewältigung neuer Arbeitsformen. Vielmehr sollte sich das ethnografische Interesse auf die weit reichende Ambivalenz einer (vormals im Kreativitätskonzept eingelagerten) ,popular agency“ richten. Es sollte

dazu beitragen, die Innenseite dieses Geschehens zu erhellen. Werden die Bewältigung unterschiedlicher Jobs, die zeitliche Koordination der verschiedenen Arbeitsaufgaben sowie das Herausschlagen von Restzeiten für das Privatleben nur als Stress oder auch als Selbstbestätigung empfunden? Entspricht der Kreativitäts-Zuschreibung von außen ein Stolz auf die eigenen Fähigkeiten und Befriedigung über das Geleistete? [...] Und es wäre sicherlich voreilig, ein solches Selbstverständnis als Illusion abzuhaken, statt nach Realien oder Potentialen zu suchen, die solch eine Selbstsicht erklären und es manchmal sogar rechtfertigen, ihm vorwärtstreibende Momente zuzuerkennen. (Warneken 2006: 125)

Diesem Forschungsauftrag kommt die Arbeitskulturforschung bereits nach, indem sie die realen Seiten der Ambivalenz von Selbstausbeutung und Selbstverwirklichung, von Armutsrisiken und Autonomiegewinnen

1 Die Kritik an Richard Floridas Konzept der kreativen Klasse richtet sich deshalb auch häufig gegen die Normativität, die es aus eben jenen Anforderungen zieht. 
beschreibt und nachvollziehbar macht (Moser 2003; Seifert/Götz/Huber 2007). Diese Forschungsperspektive kann durch Fragen nach Risikopraktiken nochmals zugespitzt werden. Für den spezifischen Bereich der urbanen Kreativwirtschaft und der darin zu beobachtenden alltagskulturellen Bedeutung von Risiko wären dann drei Untersuchungsperspektiven $\mathrm{zu}$ unterscheiden: eine akteurszentrierte, eine praxiszentrierte und eine auf die Stadtform konzentrierte Perspektive. Im Zusammenspiel dieser Ansätze können die involvierten Akteurskonstellationen, Risikopraktiken und stadtspezifischen Ausformungen von Risikobereitschaft erhoben und auf ihre Alltagsrelevanz hin untersucht werden.

\section{Akteurszentrierte Perspektive}

In einer akteurszentrierten Perspektive ist zunächst einmal von Interesse, für wen welche Risiken innerhalb des Kontexts der Kreativwirtschaft gelten? Denn die Komplexität - und die von der UNESCO attestierte große Spannbreite an Erfolgs- oder Misserfolgschancen - geht u. a. auf die historisch verankerte Vielfalt involvierter Akteursgruppen zurück. Wie lassen sich Akteursgruppen beschreiben und wie ist Risiko und Risikobereitschaft zwischen ihnen verteilt?

Die ethnografische Studie der Stadtsoziologin Silke Steets über Leipzigs Kreativwirtschaft liefert für die Beantwortung dieser Fragen eine Reihe von Ansatzpunkten (Steets 2008). ${ }^{2}$ Leipzig scheint vor wie nach der Wende eine für kreative Milieus (Metzler 2008: 42f.) ${ }^{3}$ attraktive Stadt gewesen und noch heute zu sein: Als Knotenpunkt für Netzwerke von Kreativen kann die mit der Leipziger Hochschule für Grafik und Buchkunst einher gehende Kunstszene, vor allem im Bereich Malerei, gelten, die seit Ende der 1990er Jahre über den bundesdeutschen

2 Steets rekonstruiert in dieser Ethnografie die (Vor-)Geschichte der Kreativwirtschaft in Leipzig ab den frühen 1990er Jahren über ca. 15 Jahre.

3 Mit dem Begriff der „kreativen Milieus“ untersucht die Historikerin Birgit Metzler am Beispiel des Nauwieser Viertels in Saarbrücken die Geschichte kultureller Netzwerke ab den 1970er Jahren bis heute. Interessant an diesem Fall ist die weit über das Viertel und die Stadt hinausreichende Sichtbarkeit des an politischen Zielen orientierten alternativen „kreativen Milieus", das sich aber weder nachhaltig als Zentrum von kreativwirtschaftlichen Aktivitäten etablieren konnte noch in das Bild der Stadt als solche hätte vordringen können. Metzger verweist damit auf die Diskontinuitäten kreativer Milieus und, dass ,[t] he simple presence of a bohemian, alternative milieu in a city seems not to be a sufficient condition for economic development as claimed especially by Richard Florida“. Um tiefere Einsicht über die Bedingungen von Erfolg zu erlangen, müssten deshalb auch Fälle von Misserfolg in der Kreativwirtschaft untersucht werden. 
Kontext hinaus zur Verknüpfung der Stadt mit dem globalen Kunstmarkt geführt hat (Steets 2008: 171ff.). Während dieser Bereich der Kreativwirtschaft, nicht nur in Leipzig, zu den schillerndsten zählt, was sich u. a. in der besonders großen Spannbreite der Marktchancen für die Individuen ausdrückt, sind in den frühen 1990er Jahren subkulturelle, spartenübergreifende kulturelle Netzwerke entstanden. $\mathrm{Zu}$ dieser Zeit bildeten sich diese Netzwerke bereits zu einem großen Teil aus Ost- wie Westdeutschen, denn viele der jungen AkteurInnen waren aus den alten Bundesländern zugezogen. Eine spezifische Akteurskonstellation, die der historischen Umbruchssituation geschuldet war und derjenigen im Ostteil Berlins ähnelte. Diese Spezifik beruhte auch auf der besonderen räumlichen Situation, d. h. neben den industriellen Brachen auf dem Leerstand im Wohn- und Gewerberaumbereich. Steets vollzieht in ihrer Studie „Wir sind die Stadt!“ das Werden dieser Netzwerke nach, die sich zunehmend professionalisieren und Leipzig nur zum Teil explizit auch als Identifikationsraum benennen.

Für einen der zentralen Akteure eines kulturellen Netzwerkes „komme es vor allem auf das Netzwerk an Leuten an, mit denen er Projekte realisieren kann. Und das sei in Leipzig ohne Zweifel vorhanden, aber nicht direkt an die Stadt oder den lokalspezifischen Kontext gebunden“ (Steets 2008: 185). Dass er hier dennoch schon seit zehn Jahren kontinuierlich lebe, interpretiert Steets nicht etwa mit dem Konzept der „sticky places“, die deshalb attraktiv sind, weil an ihnen ein gelungenes „Zusammenspiel zwischen Wissensmilieus [...] und lokalen Organisationen beziehungsweise institutionellen Strukturen" stattfinde (ebd.: 189). Vielmehr zeichneten sich für diese Akteure die von ihnen hergestellten/genutzten Orte durch die Vorstellung eines „nutzungsoffenen Raums [aus], eines Raums der kreative Formen der Aneignung zulässt, an dem man die ,richtigen Leute" trifft und der neuartige Formen der Kulturproduktion erlaubt“ (ebd.: 185). Deshalb schlägt Steets den Begriff der ,wohnzimmertypischen Vergemeinschaftungsform“ vor.

Hinsichtlich der Verteilung von Risiko und Risikobereitschaft liefert die Studie einige Ansatzpunkte: So verweist die von den AkteurInnen hervorgehobene geringe Anzahl von Orten, an denen sich in der Entstehungsphase immer die selben Personen getroffen hätten, zum einen auf den Pionierstatus, den sie sich rückblickend zuschreiben - und damit auch auf ihre Risikobereitschaft. Andererseits relativiert genau diese Übersichtlichkeit den Grad des Risikos für kreative Unternehmungen: die neue Situation wird dadurch in ihren Unwägbarkeiten überschaubar und kalkulierbar. Wer agiert also innerhalb dieses Feldes mit hoher Risikobereitschaft, wer mit weniger oder gar ohne? Dabei gilt es in diesem Fall zu bedenken, dass diese Situation zwar für alle gleichermaßen neu 
war. Sie war aber für Ost- und Westdeutsche unterschiedlich herbeigeführt worden (Systemwandel vor Ort vs. Systemtransfer und Ortswechsel usw.) und man ist ihr deshalb mit einem unterschiedlichen Repertoire an Alltagspraktiken begegnet; Risikobereitschaft und der Umgang mit Risiko müssen davon nachhaltig geprägt worden sein.

Die Studie zu Leipzig beschränkt sich mit Blick auf die Akteurskonstellationen nicht auf die Untersuchung der Kreativen oder UnternehmerInnen selbst. Sie bezieht die an der politischen und wirtschaftlichen Identifizierung, Steuerung und Aufwertung der Kreativwirtschaft Beteiligten ein, was für alle akteurszentrierten ethnografischen Untersuchungen gelten muss. Dazu zählen auf politischer Ebene Bundesministerien und einzelne kommunale Verwaltungen, zudem öffentliche wie private Institutionen (Handelskammern, Forschungsinstitute); darüber hinaus aber auch herausragende, impulsgebende Einzelpersonen und Medien (Friedrichs 1998). Über welche Instrumente werden hier Interessen und Akteursgruppen konstruiert (bspw. in Form von Kulturwirtschaftsberichten und der Benennung von dazu zählenden Sektoren, Institutionen, Einzelpersonen) und wer trägt welches Risiko innerhalb des damit nachweisbaren Zusammenhangs „Kreativwirtschaft"? Nicht alle Akteursgruppen dürften hier (demselben) Risiko begegnen.

\section{Praxiszentrierte Fragestellungen}

Eine empirisch praxiszentrierte Forschungsperspektive widmet sich nicht der Frage, ob Individuen und Gruppen in unterschiedlichem Maße Risikobereitschaft umsetzen, sondern wie sie dies tun und wie sie mit verschiedenen Formen von Risiko umgehen, die der KreativwirtschaftsKomplex hervorbringt. Wie wird in den einzelnen Akteurskonstellationen welches Risiko verhandelt - was schafft Anreize, was dämpft Risikobereitschaft? In welche Richtung führen Kalkulationspraktiken und -instrumente?

Im Zentrum steht dann das Herausarbeiten konkreter Praktiken, die das Verhältnis von hohem Entscheidungszwang, gesteigerter Unsicherheit und Kalkulation von Wahrscheinlichkeit des Erfolgs oder des Scheiterns bestimmen. Wie werden diese Wahrscheinlichkeiten „kalkuliert“ bzw. praktisch umgesetzt?

Obwohl Risikobereitschaft und damit einhergehende kulturelle Praktiken in der Studie von Steets nicht thematisch ausgeführt werden, zeigt die detaillierte Beschreibung des öffentlich-privaten Charakters dieser szeneförmigen Netzwerke, dass ein individuelles Risiko für eine neue Unternehmung innerhalb des jeweiligen Netzwerkes abgefedert wird. So auch für die Autorin selbst, deren Zugang zum Forschungsfeld über die 
eigene Beteiligung an Kulturproduktionen zustande kam. Diese Form der Teilnehmenden Beobachtung hatte „,den Arbeitsalltag freischaffender Kulturproduzenten und eine sehr persönliche Erfahrung des ökonomischen Überlebenskampfes“ (Steets 2008: 18) zum Gegenstand und erlaubt ein differenziertes Bild der sozialen Praktiken der „Szenevergemeinschaftung“ (ebd.: 211). Diese Praktiken erzeugen eine geringe Fallhöhe für die einzelnen Akteure, indem die Überschaubarkeit der Netzwerke beibehalten wird. Sie äußern sich darin, dass das (zahlende) $\mathrm{Pu}$ blikum einer Kulturproduktion vor allem aus den anderen Szenemitgliedern besteht und dass sie vielfältige, zeitlich begrenzte Kooperationen zwischen den einzelnen Akteuren erzeugen: „Gruppen formieren sich für eine Filmproduktion, eine Bandgründung, eine Clubshow, ein zweiwöchig betriebenes Café, eine künstlerische Intervention oder die Aufnahme eines Hörspiels und lösen sich nach Beendigung des Projektes wieder auf.“ Ausschlaggebend ist: „Als Potentialität aber existieren sie weiter: Man bleibt in Kontakt, behält unter Umständen sogar das gemeinsame Label und wenn sich ein neues Projekt ergibt, kommt man wieder zusammen oder bildet neue Kooperationen.“ (Ebd.: 17).

„Wahrscheinlichkeit“, ein Element von Risiko, wird hier positiv gewendet zur „Wahrscheinlichkeit wieder zu kooperieren“ und nicht etwa als mangelnde Planbarkeit oder Ungewissheit artikuliert. Flankiert wird diese projektförmige Risikopraxis von geringen finanziellen Investitionen und einer Mischfinanzierung des Lebensunterhalts, die aus unterschiedlichen Arbeitsverhältnissen resultiert.

Ebenfalls auf individuellen „Mischfinanzierungen“ beruht das Beispiel einer „Verkaufsplattform“ für Design in Berlin. Im Unterschied zu den bei Steets beschriebenen kulturellen Netzwerken sind die hier angewandten kooperativen Praktiken jedoch nicht spartenübergreifend und führen nicht zu einer Szenevergemeinschaftung; sie dienen allein der Kalkulation von Erfolgs- oder Misserfolgschancen.

Bei dem hier gewählten Beispiel aus einer Langzeituntersuchung zu „Kaufhausprojekten“, die sich auf Design aus Berlin spezialisiert haben, (Färber 2008: 417-425) handelt es sich um ein Kaufhaus, das als Verkaufs- und Arbeitsraum in einem Gebäude im Stadteil Friedrichshain von wechselnden KooperationspartnerInnen betrieben wird. An diesem anfangs noch vom Quartiersmanagement unterstützten Kaufhaus sind ExistenzgründerInnen anhand unterschiedlicher Praktiken beteiligt (zu Beginn noch gefördert durch das Format der Ich-AG). Das geringste finanzielle und zeitlich-räumliche Engagement gehen diejenigen ein, die sich ein Regal mieten, um ihre Waren auszustellen und zum Verkauf anzubieten. Gängistes Model ist jedoch eine kombinierte Werkstatt- und Verkaufsraumnutzung auf Grundlage eines monatlichen Mietbeitrags 
und der Abgabe von $10 \%$ der Einnahmen in die gemeinsame Kasse. Die tägliche Anwesenheit ist in keinem der beiden Modelle verpflichtend, da der Verkauf von einer Person gehandhabt werden kann, die dann die Kassen der jeweils nicht anwesenden DesignerInnen mit verwaltet und einen Abschlag für diese Tätigkeit erhält. Auf diese Weise ist die Ressource Zeit relativ frei verfügbar und wird von einigen der ca. zehn ständigen MieterInnen dahin gehend genutzt, dass sie die eigenen Waren an anderen Orten (z. B. Märkten, weitere Geschäfte oder auch in Onlineshops) anbieten - und überhaupt entwerfen und fertigen. Das finanzielle Risiko, das ein etwaiger geringer Umsatz im Kaufhaus selbst darstellen könnte, wird durch diese Mischformen ausgeglichen, indem vom Kaufhaus aus räumlich flexibel gehandelt wird. Die Fluktuation ist indes relativ groß, von den anfänglichen MieterInnen sind nach vier Jahren nur noch zwei vertreten. Viele sind „Pleite gegangen“, wie die Hauptmieterin ohne Umschweife feststellt - oder haben sich, sobald sich ein gewisser Erfolg abzeichnete, aus der Gemeinschaftsnutzung verabschiedet und ihre eigenen Geschäfte eröffnet.

Insgesamt zeichnet sich das Projekt zum einen durch festgelegte Praktiken aus, die zu einer Minimierung der Wahrscheinlichkeit des Scheiterns beitragen sollen: das Teilen von Raum und Zeit, die anteilige Verteilung von Verantwortung und Ressourcen. Darüber hinaus bleibt aber jeder/m einzelnen überlassen, sein/ihr Geschäft über das Kaufhaus hinaus zu betreiben. Dem unternehmerischen Risiko wird so gesehen temporär gemeinschaftlich und räumlich flexibel begegnet. Die Konzentration auf diese relative Absicherung hängt von den individuellen Ressourcen ab, das jeweilige Mischverhältnis zeichnet sich als Risikobereitschaft ab.

Langfristige Teilnehmende Beobachtung mit der Perspektive „Risiko und Risikobereitschaft" wird genaueren Aufschluss darüber geben, wie sich Planung unter Bedingungen der Planungsunsicherheit vollzieht, wie Absicherung unter Bedingungen der Unsicherheit und berechenbarer Risiken geschieht.

\section{Form- und stadtzentrierte Fragestellungen}

Schließlich stellt sich die Frage, in welcher Form Risiko in der Kreativwirtschaft neben seinen ökonomischen Formaten (von privaten Krediten bis zum Risikokapital) auftritt: Welchen kulturellen Ausdruck finden mangelnde Planbarkeit, (fehlende) ökonomische und soziale Absicherung oder der Gewinn/Verlust an symbolischem Kapital, den die Wahrscheinlichkeit des Erfolgs oder des Scheiterns in der Kreativwirtschaft 
mit sich bringen kann? Wenn „Selbstbestimmung und soziale Deklassierung [...] in der Kreativwirtschaft offenbar untrennbar miteinander verknüpft" sind (Manske 2008: 52; 2007), in welcher Form und in welchem Medium artikuliert sich das Paradox des Risikos im Alltag der AkteurInnen: in Form von Gefahr, Absicherung oder „Risikofreude“, in ironischen Selbstreflexionen, Rationalisierungen, die bspw. ohne digitale Medien nicht zu erlangen wären?

Ohne den Bezug zur akteurs- und praxiszentrierten Perspektive lässt sich die Frage der Form sicher nicht differenziert beantworten. Und auch eine stadtanthropologische Perspektive muss hier zu Rate gezogen werden, die Risikoformate, Praktiken und Akteurskonstellationen hinsichtlich ihrer Einbettung in die „Eigenlogik“ der jeweiligen Stadt erklärt (Berking/Löw 2008).

Risiko als kalkulierbare Möglichkeit des Erfolgs und des Scheiterns hat sich in Berlin als Topos ganz explizit Eingang in das Feld der urbanen Kreativwirtschaft verschafft: in öffentlichen Darstellungen der städtischen Wirtschaftslage und in Role-models. Dass Kulturproduktion in Berlin heute nämlich als florierende Kreativwirtschaft wahrgenommen wird, hat sie auch einer Vielzahl diskursiver Anstrengungen zu verdanken, die Wahrscheinlichkeiten des Erfolgs und des Scheiterns thematisieren und u. a. dadurch kalkulierbar machen.

Eine Analyse der diskursiven Reevaluierung und Einbettung einer als stagnierend wahrgenommenen wirtschaftlichen Gesamtlage des Landes Berlin und einer gleichzeitig dynamischen Kulturproduktion von Seiten öffentlicher und privater Institutionen, wie dem Senat und der Industrie und Handelskammer (IHK), zeigt eines: Alle Akteure, die sich mit der wirtschaftlichen Lage Berlins als Ganzes auseinander setzen müssen, können die Spannung zwischen ökonomischer Ödnis einerseits und florierender Kultur und Kulturproduktion andererseits, nur als Gegensatz darstellen. Gemäß der unterschiedlichen Zuständigkeiten, Positionen und Kontexte wird diese Spannung zwar unterschiedlich aber stets als inkommensurabler Gegensatz formuliert. So schreibt die IHK Berlin in einer Pressemitteilung zur Gesamtlage des industriellen Sektors:

Die Industrie in Berlin ist anders, aber sie ist quicklebendig. Sie ist geschrumpft aber mit ihren Produkten ist sie auf den Weltmärkten wettbewerbsfähig. [...] Die Industrie beschäftigt weniger Mitarbeiter, deren Qualifikation und durchschnittliches Einkommen sind aber höher. ${ }^{4}$ 
Wirtschaftssenator Harald Wolf (Die Linke) stellt als „Grunddilemma, das wir an unserem Standort haben“ fest: „Realität und Potenzial klaffen in Berlin weit auseinander.“ (Waldermann 2005) Bürgermeister Klaus Wowereit (SPD) spricht laut Medienberichten auf einer Dienstreise nach London von ,den maroden Finanzen und wirtschaftlichen Strukturen, die man nicht gerade als gesund bezeichnen könne. Aber Berlin sei eine junge, ,unfertige Stadt", in der man mitmischen könne." Für dieses Dilemma findet er das äußerst medienwirksame Bild: Berlin sei „Arm aber sexy“ (Thibaut 2003). Die Wahrscheinlichkeit ökonomischen Scheiterns tritt in dieser anthropomorphen Wendung letztendlich als Gegensatz zu symbolischem Erfolg auf, beides scheint unvermeidbar aber anknüpfbar und möglicherweise auch verwertbar.

Mit dem Instrument des Kulturwirtschaftsberichts, der 2004 von der „Berliner Initiative für Kreativwirtschaft“ angestoßen ${ }^{5}$ und 2005 in $\mathrm{Zu}$ sammenarbeit mit den damaligen Senatsverwaltungen für Wirtschaft, Arbeit und Frauen sowie für Wissenschaft, Forschung und Kultur umgesetzt wurde, ließ sich nun ein Sektor aus der desolaten Gesamtlage „herausschneiden" - die Kreativwirtschaft. Damit konnten nicht nur AkteurInnen, Netzwerke, vermittelnde Formate (Messen, Großhandel), öffentliche Institutionen und Ereignisse identifiziert werden, die das Feld der nun „offiziellen“ Kreativwirtschaft absteckten. Gleichzeitig war auch ein Instrument geschaffen, mit dem sich fortan eindeutige Zahlen über die Produktivität dieses Sektors liefern lassen. Innerhalb der gesamt gesehen schlechten Wirtschaftslage ließ sich die Dynamik dieses Sektors fortan repräsentieren.

Anstelle des unerklärlichen Widerspruchs tritt nun eine Darstellung, in der die Materialisierung des Scheiterns alter Industrien in Gestalt des industriellen Brachlands zur Voraussetzung für Kulturproduktion und damit für die Kreativwirtschaft wird. Der sich seit den 1990er Jahren zunehmend etablierende Topos des Leerstands/der Brache als Freiraum (Fezer/Wieder 2004: 80), der in der Debatte um die schrumpfenden Städte als Strukturmerkmal aufgegriffen wurde (Oswalt 2004), hat den Ansatzpunkt für eine Semantik geboten, in die ökonomischer Stillstand und eine wachsende Kreativwirtschaft als Kausalzusammenhang eingeschrieben werden konnten: nicht ,aber“, sondern „weil“. Auf diese Weise ist auch „Risiko“ angesprochen, denn diese Rhetorik lagert die Wahr-

5 Der erste Kulturwirtschaftsbericht konnte sich in seinen Wachstumsanalysen auf zwei Berichte zu Wirtschaft und Kultur von 1992 und 2002 beziehen. 
scheinlichkeit des Scheiterns in die alten Industrien aus und bietet mit Verweis auf die bereits erfolgreiche und nun messbare Kulturproduktion einen Ansporn für die (Risiko-)Bereitschaft, sich auf diesen Wirtschaftszweig einzulassen. Dieser Logik folgend und ebenfalls von offizieller Seite unterstützt hat die Dokumentation „Urban pioneers. Stadtentwicklung durch Zwischennutzung“ das dafür notwendige Profil „urbaner Pioniere" skizziert (Overmeyer 2007). Sie ist damit ein weiterer Baustein, der zur individuellen Identifizierung mit diesem Modus der Risikobereitschaft beiträgt und Aufschluss über die veränderte Wahrnehmung und Bewertung risikobehafteter unternehmerischer Kulturproduktion im Stadtraum gibt.

Obwohl die Brachen und Leerräume auch in Berlin knapper werden und die Mieten spürbar steigen, hat sich dieses Bild auch aufgrund der oben beschriebenen Verschiebung der Rhetorik etabliert: Es basiert zum Teil auf noch immer realisierbaren Erfahrungen, zum Teil gehören diese aber schon der Vergangenheit an; es wird als grundlegendes und modellbildendes Paradox in die kulturpolitische Debatte hineingetragen (Göhler 2006: 201f.) oder - von „urbanen Pennern“ über die „,digitale Bohème“ bis zu den „urbanen Pionieren“ (Bunz 2006; Friebe/Lobo 2006; Overmeyer 2007) - in szenewirksame Role-models von Risikobereitschaft übersetzt. Diese Bilder führen ein Eigenleben, sie eilen der Stadt voraus und erzielen entsprechende Effekte (Rehberger 2009). ${ }^{6}$ Mit Blick auf die Attraktivität Berlins für Kreative und deren Risikobereitschaft legen diese Bilder eine hohe Wahrscheinlichkeit, Fuß fassen zu können, nahe und ermutigen zu einer entsprechenden Entscheidung, sich in dem wirtschaftlich sehr schwachen Umfeld niederzulassen.

\section{Ausblick}

Das Sich-Einrichten im Risiko der Kreativwirtschaft, die häufig auch geäußerte Zufriedenheit über den Grad an Selbstverwirklichung im Kontext entgrenzter Arbeit und eines risikoreichen Lebens von Seiten der AkteurInnen - und sei sie ein Schutzmechanismus - müssen empirisch ernst genommen werden. Denn eine ethnografisch-kulturwissenschaftliche Forschungsperspektive hat zum Ziel

6 So verwies jüngst der Vizepräsident der Frankfurter Städelschule auf die vergleichbar niedrigen Lebenshaltungskosten in Berlin, um die Stadt Frankfurt zu mahnen, ,ihre“ Kreativen nicht nur (sehr gut) auszubilden, sondern sie auch durch die Ermöglichung ähnlicher Lebensbedingungen in der Stadt zu halten. 
über die Vor- und Nachteile dieser Beweglichkeit zu informieren. Es bedeutet andererseits, diese Akteurssicht nicht auf Meinungsäußerungen und die Erhebungsmethode nicht auf Interviews zu reduzieren, sondern sich durch Beobachtung und Datenanalyse ein eigenes Bild von den Ursachen und Folgen einschließlich der Langzeitfolgen - flexiblisierter Arbeits- und Lebensweisen zu machen. Nur dann ist es möglich, etwas über Situationsverklärungen und illusionäre Hoffnungen, aber auch über eventuelle unnötige Befürchtungen oder das Übersehen von Flexiblisierungschancen herauszubekommen, was dann zum Eruieren der kulturellen Hintergründe für Fehleinschätzungen oder selektive Wahrnehmung weiterführen kann. (Warneken 2006: 128f.)

Mit dem Forschungsfeld Risiko innerhalb der Kreativwirtschaft ist außerdem eine Beobachtungsperspektive eingezogen, die Aufschluss darüber geben kann, wie Entscheidungen, Unsicherheiten und Wahrscheinlichkeiten individuell in ein alltagstaugliches Verhältnis gebracht werden können. Darüber hinaus eröffnet diese Perspektive aber auch die Möglichkeit, das Abwägen eines individuell passenden Verhältnisses der Risikoelemente nicht als individuell bestimmt zu verstehen, sondern die kollektiven, gesellschaftlichen Anteile darin zu erkennen. In dieser Hinsicht kann ethnografisch-kulturwissenschaftliches Wissen über kulturelle Praktiken im Zusammenhang von Risiko und Risikobereitschaft (alltagstaugliche Verhältnisse zwischen Entscheidung, Unsicherheit und Wahrscheinlichkeit herzustellen) auch für Fragen der „Steuerung“ von Interesse sein. Denn es kann zu einer Stärkung dieser Praktiken auf der Grundlage von geteiltem Wissen beitragen, aus individuellen Lagen, kollektive Interessen werden zu lassen. Während das unternehmerische Selbst nämlich u. a. deshalb alleine dasteht, weil es in einem ständigen Abgrenzungsdruck der „Norm der Individualität“" entsprechen müsse (Bröckling 2007: 68), ließen sich hier Beobachtungen darüber auswerten, wie individuelle Risikobereitschaft in gemeinsame Interessen übersetzt werden kann und daraus Praktiken der Interessensvertretung werden könnten. Dass die bestehenden Instrumente, wie bspw. die Künstlersozialkasse oder gewerkschaftliche Beratungsleistungen für Freiberufliche, zum „Abbau von Lebensrisiken“ (Friebe/Lobo 2006: 285) für einen erweiterten Kreis Betroffener nicht ausreichen werden, liegt nahe; ob das Modell der Kulturgesellschaft realisierbar ist, muss sich noch erweisen, die Aufgaben dafür sind skizziert (Göhler 2006). Szenen, temporäre Kooperationen, Netzwerke sind bis heute nur selten in der Lage auch politisch nachhaltige Effekte für ihre Belange zu erreichen. Eine ethnografisches Wissen einbeziehende „Steuerung“ der Alltagssituation von Kreativen in der Kreativwirtschaft könnte aber in diese Richtung wirken. 


\section{Literatur}

Beck, Ulrich (2007): Weltrisikogesellschaft. Auf der Suche nach der verlorenen Sicherheit, Frankfurt/Main: Suhrkamp.

Berking, Helmuth/Löw, Martina (Hg.) (2008): Die Eigenlogik der Städte. Neue Wege für die Stadtforschung, Frankfurt/Main: Campus Verlag.

Bröckling, Ulrich (2007): Das unternehmerische Selbst. Soziologie einer Subjektivierungsform, Frankfurt/Main: Suhrkamp.

Bunz, Mercedes (2006): „Meine Armut kotzt mich an“, In: zitty 4: S. 17-19.

Deutscher Bundestag (2007): Schlussbericht der Enquête-Kommission „Kultur in Deutschland“, Drucksache 16/7000, 11.12.2007.

Färber, Alexa (2008): „Flourishing Cultural Production in Economic Wasteland: Three Ways of Making Sense of a Cultural Economy in Berlin at the Beginning of the Twenty-first Century“. In: Heßler, Martina/Zimmermann, Clemens (Hg.), Creative Urban Milieus, Frankfurt/Main: Campus Verlag, S. 409-428.

Fesel, Bernd/Söndermann, Michael (2007): Culture and Creative Industries in Germany, Bonn: German Commission for UNESCO.

Fezer, Jesko/Wieder, Axel John (2004): „Raum begrenzter Möglichkeiten - Stadtentwicklung in Berlin nach 1989“. In: Komplex Berlin. Katalog der 3. Berlin Biennale. Köln: Walther König, S. 73-82.

Florida, Richard (2002): The Rise of the Creative Class. And How It's Transforming Work, Leisure, Community and Everyday Life, New York: Basic Books.

Friebe, Holm/Lobo, Sascha (2006): Wir nennen es Arbeit. Die digitale Bohème oder: Intelligentes Leben jenseits der Festanstellung, München: Heyne.

Friedrichs, Jürgen (1998): „Soziale Netzwerke und die Kreativität einer Stadt". In: Albrecht Göschel/Volker Kirchberg (Hg.), Kultur in der Stadt: stadtsoziologische Analysen zur Kultur, Opladen: Leske + Budrich, S. 145-163.

Göhler, Adrienne (2006): Verflüssigungen. Wege und Umwege vom Sozialstaat zur Kulturgesellschaft, Frankfurt/Main: Campus Verlag.

Hess, Sabine/Moser, Johannes (Hg.) (2003): Kultur der Arbeit - Kultur der neuen Ökonomie. Kulturwissenschaftliche Beiträge zu neoliberalen Arbeits- und Lebenswelten, Kuckuck. Notizen zur Alltagskultur, Sonderband 4, Graz: Institut für Volkskunde und Kulturanthropologie.

Heßler, Martina (2007): Die kreative Stadt. Zur Neuerfindung eines Topos, Bielefeld: transcript Verlag. 
Heßler, Martina/Zimmermann, Clemens (Hg.) (2008): Creative Urban Milieus. Historical Perspectives on Culture, Economy, and the City, Frankfurt/Main: Campus Verlag.

IHK Pressemitteilung, Berlin 7.6.2002.

Krätke, Stefan: Medienstadt (2002). Urbane Cluster und globale Zentren der Kulturproduktion, Opladen: Leske + Budrich.

Kulturwirtschaft in Berlin: Entwicklung und Potenziale, Hg. von Senatsverwaltung für Wirtschaft, Arbeit und Frauen in Berlin, Senatsverwaltung für Wissenschaft, Forschung und Kultur. Berlin 2005.

Kokot, Waltraud (Hg.) (2000): Kulturwissenschaftliche Stadtforschung: Eine Bestandsaufnahme, Berlin: Reimer Verlag.

Lange, Bastian: Die Räume der Kreativszenen. Culturepreneurs und ihre Orte in Berlin, Bielefeld: transcript Verlag 2007.

Manske, Alexandra (2008): „Prekarisierung auf hohem Niveau oder: Risikolage Kreativarbeit“. In: Heinricht-Böll-Stiftung (Hg.), Kreativen:Wirkung. Urbane Kultur, Wissensökonomie und Stadtpolitik, Berlin: Heinrich Böll Stiftung: S. 48-52.

Manske, Alexandra (2007): Prekarisierung auf hohem Niveau. Eine Feldstudie über Allein-Unternehmer in der IT-Branche, München: Rainer Hampp Verlag.

Metzler, Birgit (2008): „Creative Milieus: Concepts and Failures“. In: Heßler, Martina/Zimmermann, Clemens (Hg.), Creative Urban Milieus, Frankfurt/Main: Campus Verlag, S. 379-408.

O’Connor, Justin (2005): „Creative Exports: Taking ,Cultural Industries“ to St Petersburg“". In: International Journal of Cultural Policy 11, S. 45-59.

Oswalt, Philipp (2004): Schrumpfende Städte. Band 1: Internationale Untersuchung, Ostfildern-Ruit: Hatje Cantz Verlag.

Overmeyer, Klaus/Berlin Senatsverwaltung für Stadtentwicklung (Hg.) (2007): Urban Pioneers. Stadtentwicklung durch Zwischennutzung, Berlin: Jovis Verlag.

Rehberger, Tobias (2009): „Mein 2009“. In: Journal Frankfurt 1, S. 46.

Schmidt-Lauber, Brigitta (2007): „Feldforschung. Kulturanalyse durch teilnehmende Beobachtung“. In: Göttsch, Silke/Lehmann, Albrecht (Hg.), Methoden der Volkskunde. Positionen, Quellen, Arbeitsweisen der Europäischen Ethnologie, Berlin: Reimer Verlag, S. 219248.

Schmidt-Semisch, Henning (2004): „Risiko“. In: Bröckling, Ulrich/ Krasmann, Susanne/Lemke, Thomas (Hg.), Glossar der Gegenwart, Frankfurt/Main: Suhrkamp, S. 222-227. 
Schwanhäußer, Anja (2008): „Raumästhetik und Raumlabore: Zur kulturellen Einbettung kreativen Schaffens im Neuen Berlin“. In: Zeitschrift für Kulturwissenschaften 1, S. 114-116.

Scott, Allen J. (2000): The Cultural Economy of Cities. Essays on the Geography of Image-Producing Cities, London: Sage.

Seifert, Manfred/Götz, Irene/Huber, Birgit (Hg.) (2007): Flexible Biografien. Horizonte und Brüche im Arbeitsleben der Gegenwart, Frankfurt/Main: Campus Verlag.

Stadt und Kreativität: „Ein Konzept als Perspektive und Effekt. Eine Diskussion zwischen Stadtforschenden“. In: Zeitschrift für Kulturwissenschaften 1 (2008), S. 106-132.

Steets, Silke (2008): „Wir sind die Stadt!“”: Kulturelle Netzwerke und die Konstitution städtischer Räume in Leipzig, Frankfurt/Main: Campus Verlag.

Thibaut, Matthias (2003): „Arm, aber sexy: Wowereit warb in London für Berlin“. In: Der Tagesspiegel 4.12.2003.

Vogt, Sabine (2005): Clubräume - Freiräume, musikalische Lebensentwürfe in den Jugendkulturen Berlins, Kassel: Bärenreiter Verlag.

Waldermann, Anselm (2005): „Unternehmer glauben an die Zukunft“. In: Der Tagesspiegel 24./25.3.2005.

Warneken, Bernd Jürgen (2006): Die Ethnographie popularer Kulturen. Eine Einführung, Wien/Köln/Weimar: Böhlau Verlag. 